\title{
Regression of cardiomyocyte hypertrophy in SHR following chronic inhibition of the $\mathrm{Na}^{+} / \mathrm{H}^{+}$exchanger
}

\author{
María C. Camilión de Hurtado ${ }^{1}$, Enrique L. Portiansky ${ }^{1}$, Néstor G. Pérez ${ }^{1}$, \\ Oscar R. Rebolledo, Horacio E. Cingolani*,1 \\ Centro de Investigaciones Cardiovasculares, Facultad de Ciencias Médicas, Facultad de Ciencias Veterinarias, Universidad Nacional de La Plata, \\ 60 y 120 (1900) La Plata, Argentina
}

Received 16 August 2001; accepted 9 November 2001

\begin{abstract}
Objective: Experiments were performed to examine the effect of chronic inhibition of the $\mathrm{Na}^{+} / \mathrm{H}^{+}$exchanger isoform-1 (NHE-1) on cardiac hypertrophy of spontaneously hypertensive rats (SHR). Methods: SHR were orally treated during 1 month with two different doses $(0.3$ and $3.0 \mathrm{mg} / \mathrm{kg}$ / day) of the NHE-1 inhibitor, cariporide, or nifedipine $(10.0 \mathrm{mg} / \mathrm{kg} /$ day $)$. Results: The two doses of cariporide did not differ in their effects after 1 month of treatment, since both induced a slight decrease in systolic blood pressure (SBP) of $\sim 6$ $\mathrm{mmHg}$ and regression of the heart weight to body weight ratio $(\mathrm{mg} / \mathrm{g})$ from $3.28 \pm 0.05$ to $3.04 \pm 0.05(0.3 \mathrm{mg}) \mathrm{and} 2.99 \pm 0.10(3.0 \mathrm{mg}$, $P<0.05)$. Nifedipine, given for the same period, produced similar reduction in the hypertrophy index $(3.03 \pm 0.05)$, but with a much greater decrease in arterial pressure $(35.6 \pm 7.4 \mathrm{mmHg})$. Chronic treatment with cariporide induced a complete regression of the augmented cross sectional area of left ventricular myocytes without significant changes in collagen content, serum procollagen 1 propeptide levels or myocardial distensibility. Conclusions: NHE inhibition represents a novel approach to induce regression of pathological hypertrophy of the heart. The finding can be rationalized mechanistically by previous in vitro studies suggesting a role of the NHE in the development of myocardial hypertrophy. (c) 2002 Elsevier Science B.V. All rights reserved.
\end{abstract}

Keywords: Fibrosis; Hypertension; Hypertrophy; Ion exchangers; $\mathrm{Na} / \mathrm{H}$ exchanger

\section{Introduction}

Mechanical stretch is one of the most important stimuli leading to cardiac hypertrophy. One of the earliest events detected after stretching adult cardiac muscle is an enhanced activity of the $\mathrm{Na}^{+} / \mathrm{H}^{+}$exchanger (NHE) [1]. The stretch-induced activation of the NHE is the result of an autocrine/paracrine chain of events involving the release of angiotensin II (Ang II) and endothelin (ET) [2-4]. The release of these transmitters by stretching isolated neonatal myocytes has been recognized since the seminal paper by

\footnotetext{
*Corresponding author. Tel.: +54-221-483-4833; fax: +54-221-4255861.

E-mail address: cicme@infovia.com.ar (H.E. Cingolani).

${ }^{1}$ Established Investigators of Consejo Nacional de Investigaciones Científicas y Técnicas (CONICET), Argentina.
}

Sadoshima et al. [5]. Experiments performed in isolated neonatal rat cardiac myocytes have further demonstrated that ET-1 could also be detected in the cardiomyocyte culture medium $10 \mathrm{~min}$ after stretch [6]. The activation of MAP kinases and protein synthesis can be attenuated by inhibition of the NHE when promoted by stretch, but not after the addition of Ang II or ET-1 [7]. Furthermore, an enhanced activity of the NHE in the hypertrophied myocardium of the SHR [8,9] and the normalization of NHE activity after enalapril-induced regression of cardiac hypertrophy [10] have been reported.

The mechanism by which mechanical stretch transmits signals to the nucleus is not completely clarified. It remains possible that myocardial stretch activates kinase cascades, which lead to cardiac hypertrophy and activation

Time for primary review 22 days. 
of the NHE in a parallel and independent fashion. If so, the enhanced activity of the NHE would be an epiphenomenon of the hypertrophy and chronic inhibition of the NHE should not be accompanied by a regression of myocardial hypertrophy. In order to shed light on this crucial issue, experiments were performed in SHR in which the effect of chronic inhibition of the NHE on myocardial hypertrophy was assessed.

\section{Methods}

Experiments were conducted in accordance with the Guide for the Care and Use of Laboratory Animals (US Department of Health and Human Service) with male SHR and normotensive Wistar (NT) rats. From the age of 3 months, systolic blood pressure (SBP) was determined by the tail-cuff method [11]. At the age of 4 months, mean SBP values were $121 \pm 1(\mathrm{NT}, n=41)$ and $177 \pm 1 \mathrm{mmHg}$ (SHR, $n=53$ ). At this time, animals of each strain were randomly assigned to one of the following treatments: (a) $0.3 \mathrm{mg} / \mathrm{kg} /$ day cariporide (specific NHE-1 inhibitor); (b) $3.0 \mathrm{mg} / \mathrm{kg} /$ day cariporide; (c) $10.0 \mathrm{mg} / \mathrm{kg} /$ day nifedipine; (d) no drug (control untreated group) and (e) sacrifice to assess the degree of cardiac hypertrophy at the time of treatment initiation. While cariporide was dissolved directly in the drinking water, nifedipine was orally administered in a single daily dose. The concentration of cariporide in the drinking water was adjusted according to weekly measurements of BW and daily records of water intake to match with the selected dosages. After 1 month, the rats were euthanized under ether anesthesia. Papillary muscles isolated from the left ventricle were used to assess myocardial stiffness. After trimming off all adjacent connective tissue and the atria, the ventricles were blotted and weighed (HW) to determine the level of cardiac hypertrophy by $\mathrm{HW}$ normalized to $\mathrm{BW}$ ( $\mathrm{HW} / \mathrm{BW}$ ratio, in $\mathrm{mg} / \mathrm{g}$ ). The ventricular tissue was fixed in buffered formaldehyde $10 \%$ for $24 \mathrm{~h}$. Coronal sections obtained from the equator of the left ventricle were prepared for conventional histological techniques. Five- $\mu \mathrm{m}$-thick paraffin-embedded sections were stained either with Mallory's phosphotungstic acid hematoxylin (PTAH) method or a trichromic technique including Gill haematoxylin, xylidine punceau and picric acid to differentiate fibrils and delineate cell borders [12]. The trichrome Van Gieson stain technique was used for determining collagen in the muscle tissue [12]. All the stained sections were observed under the microscope (Olympus BX-50, Tokyo, Japan) and the images were captured using an analog video camera. Images were digitized and processed by a computer morphometry program (Image-Pro Plus for Windows 95/ 98 v4.5-Media Cybernetics, Silver Spring, MA, USA). The resolution of the microscopic images was of $640 \times 480$ pixels with a spatial calibration yield of $0.63 \mathrm{~mm}^{2} /$ pixel for determining collagen volume fraction (CVF) or 0.32 $\mathrm{mm}^{2} /$ pixel for determining the cross-sectional area of cardiac muscle cells. Fifty cross-sectioned cardiac muscle fibers were counted in at least 10 images obtained from each left ventricle. Only round to ovoid cells with visible round nucleus were considered for CSA measurements. Each cell was individually traced and its cross sectional area directly determined. CVF was calculated as the sum of all connective tissue areas of the coronal sections, divided by the total surface of the section. Perivascular collagen was excluded from this measurement. To separate the stained tissues from other objects, a color segmentation was applied. The investigator responsible for the morphological analysis was blinded as to each experimental group.

To assess myocardial stiffness, isolated left ventricular papillary muscles were mounted in a horizontal perfusion chamber between a force transducer (AEM801 SensorOne) and a fixed hook for isometric records. The muscles were superfused at a constant rate $(5 \mathrm{ml} / \mathrm{min})$ with a $\mathrm{CO}_{2} /$ $\mathrm{HCO}_{3}^{-}$-buffered solution containing (mmol/1) $128.3 \mathrm{NaCl}$, $4.5 \mathrm{KCl}, 1.35 \mathrm{CaCl}_{2}, 0.35 \mathrm{NaH}_{2} \mathrm{PO}_{4}, 20.23 \mathrm{NaHCO}_{3}, 1.05$ $\mathrm{MgSO}_{4}, 11.0$ glucose, equilibrated with $5 \% \mathrm{CO}_{2} / 95 \% \mathrm{O}_{2}$ ( $\mathrm{pH}$ 7.4). The preparations were stimulated by square pulses of $5 \mathrm{~ms}$ duration, at a voltage $\sim 20 \%$ higher than the threshold. After mounting, the values of muscle slack length $\left(L_{0}\right)$, thickness and width were determined with a reticle in the eyepiece of an SZ30 Olympus Zoom stereomicroscope set at a total magnification of $\times 30$. The muscles were progressively stretched to the length at which they developed the maximal twitch force $\left(L_{\max }\right)$ in steps of $10 \%$ of $L_{0}$. The force was normalized to the cross sectional area of each muscle, which was calculated multiplying thickness by width and corrected by a factor of 0.75 assuming an ellipsoidal shape. The stress-strain relationship for each muscle was fitted to an exponential equation. From the fitted curves, fixed values of strains $\left(\Delta L / L_{\max }\right)$ were interpolated in each experiment.

To determine serum levels of carboxyterminal propeptide of procollagen type I (PIP), blood samples were obtained at the time of euthanasia and allowed to clot spontaneously. After centrifugation, serum samples were separated and stored at $-70{ }^{\circ} \mathrm{C}$ until assayed. Serum PIP was determined by radioimmunoassay with a commercial assay kit (Orion Diagnostica, Finland). The lower detection limit (method sensitivity) was $1.2 \mu \mathrm{g} / 1$.

\subsection{Statistics}

Data were expressed as mean \pm S.E.M. One-way ANOVA and two-way ANOVA were used to compare differences, as appropriate. Dunnett was used as post hoc test. $P$ values $<0.05$ were considered to indicate significant differences. 


\section{Results}

SHR and their normotensive (NT) control rats were treated orally with two doses of the specific inhibitor of the NHE-1, cariporide, during 1 month. Fig. 1 shows the SBP values during this time. A slight decrease in arterial pressure following the treatment was detected in the SHR, and no effect was evident in the NT rats. The two doses of the inhibitor did not differ in their effect on blood pressure. On average, the value of systolic arterial pressure at the end of the treatment period was $\sim 6 \mathrm{mmHg}$ lower in the treated SHR than in the untreated animals. We did not explore the mechanism involved in this slight effect on SBP in the SHR rats. We speculate that the effect of the NHE inhibitor on cell growth might decrease wall thickness in resistance vessels of SHR [13].

The degree of cardiac hypertrophy was assessed by the $\mathrm{HW} / \mathrm{BW}$ ratio. Fig. 2 shows the decrease in $\mathrm{HW} / \mathrm{BW}$ ratios induced by treating $\mathrm{SHR}$ with cariporide, at both dosages used, either when comparison was made with age-matched untreated SHR or with 4 months old SHR. Chronic treatment with cariporide for 1 month did not modify the $\mathrm{HW} / \mathrm{BW}$ ratio in NT rats at any of the dosages used. In cariporide-treated $\mathrm{NT}, \mathrm{HW} / \mathrm{BW}(\mathrm{mg} / \mathrm{g})$ ratio were $2.47 \pm 0.14(n=4)$ and $2.56 \pm 0.10(n=7)$ after 1 -month treatment with the low- and high-dosage, respectively. These values did not differ from the $\mathrm{HW} / \mathrm{BW}$ determined in untreated NT rats at the age of treatment initiation

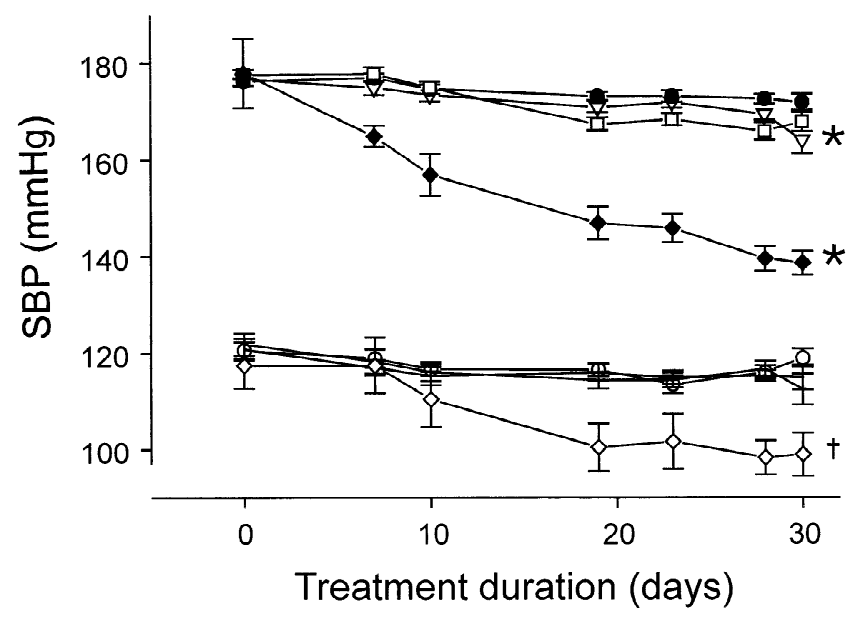

Fig. 1. SBP values in untreated and treated NT and SHR rats. -0(untreated SHR, $n=17)$; $-\nabla-($ low-dose cariporide-treated SHR, $n=15$ ); $-\square$ - (high-dose cariporide-treated SHR, $n=6$ ), - $\bullet$ - (nifedipine-treated SHR, $n=6$ ), -O- (untreated-NT, $n=14$ ); - - (low-dose cariporide-treated NT, $n=9$ ), $\cdots$ (high-dose cariporide-treated NT, $n=8$ ) and $-\downarrow$ (nifedipine-treated NT, $n=6$ ). Nifedipine treatment reduced SBP by $\sim 40$ $\mathrm{mmHg}$ in SHR and by $\sim 15 \mathrm{mmHg}$ in NT. By contrast, cariporide, at both dosages used, only in the SHR caused a slight but statistically significant decrease of SBP. *Indicates $P<0.05$ between treated-SHR groups vs. untreated-SHR (ANOVA); ${ }^{\dagger}$ indicates $P<0.05$ between nifedipine-treated and untreated NT rats (ANOVA).

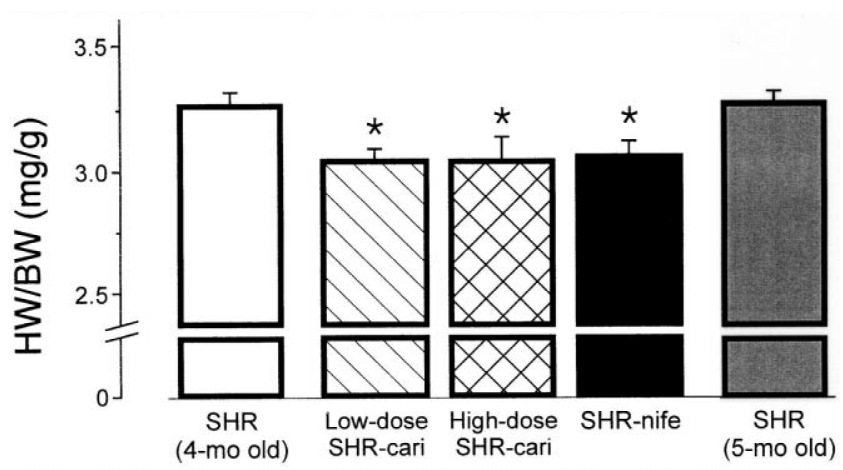

Fig. 2. Effect of NHE-1 inhibition on cardiac hypertrophy in SHR. Cardiac hypertrophy was assessed by $\mathrm{HW} / \mathrm{BW}$ ratio in 4 months old untreated SHR $(n=10)$; low-dose $(0.3 \mathrm{mg} / \mathrm{kg} /$ day $)$ cariporide-treated SHR (Low-dose SHR-cari, $n=15)$; high dose $(3.0 \mathrm{mg} / \mathrm{kg} /$ day) cariporide-treated SHR (High-dose SHR-cari, $n=6$ ); nifedipine-treated SHR (SHR-nife, $n=6)$ and 5 months old untreated SHR $(n=17)$ rats. Cariporide, at both dosages used, decreased cardiac hypertrophy in SHR either compared with $\mathrm{HW} / \mathrm{BW}$ values at the time of treatment initiation or of age-matched untreated SHR rats. Interestingly, cariporide produced about the same decrease in cardiac hypertrophy as nifedipine, despite a much lesser decrease in SBP. *Indicates $P<0.05$ between treated and untreated groups (ANOVA).

$(2.51 \pm 0.08, n=10)$ nor from age-matched untreated NT rats $(2.50 \pm 0.09, n=7)$.

Chronic NHE-1 inhibition induced a slight decrease in arterial pressure together with a partial regression of the myocardial hypertrophy, thus making it interesting to compare these two effects, namely the decrease in arterial pressure and the decrease in myocardial hypertrophy induced by the NHE-1 inhibitor, with those induced by a primary antihypertensive pharmacological intervention such as nifedipine blockade of calcium channels. Since the effect of dihydropyridines on hypertensive myocardium is well established [14-18] and this study was particularly focused on the action of NHE-1 blockade, the experiments with nifedipine were aimed only to compare the relationship between the reduction in SBP and the degree of cardiac hypertrophy regression achieved by both treatments. A similar decrease in the degree of hypertrophy was obtained with cariporide and nifedipine (Fig. 2) but with an approximately sixfold greater decrease in SBP after nifedipine-treatment (Fig. 1). The decrease in arterial pressure was only $5.7 \pm 1.7$ and $5.6 \pm 1.6 \mathrm{mmHg}$ with the low and high dose of cariporide, respectively, and $35.6 \pm 7.4 \mathrm{mmHg}$ with nifedipine $(P<0.05)$.

Fig. 3 shows the mean cross sectional area (CSA) of left ventricular cardiomyocytes in the myocardium of untreated age-matched NT and SHR, and cariporide-treated (lowand high-dose) SHR rats. A decrease in cardiomyocyte CSA by $\sim 30 \%$ was detected in the myocardium of the SHR after the 1-month treatment with the NHE-1 inhibitor at both dosages. The CSA of the cardiomyocytes in cariporide-treated SHR was not different from that of untreated age-matched NT rats. 


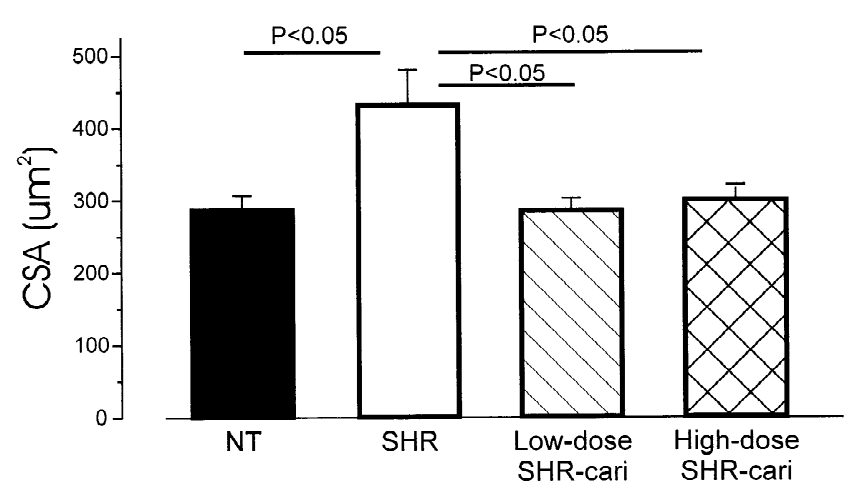

Fig. 3. Cross sectional area (CSA) of left ventricular cardiomyocytes. Mean values of CSA determined in age-matched untreated NT (closed bar, $n=4$ ), untreated SHR (open bar, $n=4$ ), low-dose cariporide-treated SHR (hatched bar, $n=5$ ) and high-dose cariporide-treated SHR (crosshatched bar, $n=4$ ) rats are shown. In SHR, NHE-1 inhibition reduced the CSA of the cardiomyocytes to a value not different from that found in NT rats.
Fig. 4 shows representative photomicrographs of left ventricular myocardium from untreated age-matched NT and SHR, and cariporide-treated (low- and high-dose) SHR rats. An enlarged CSA is observed in the myocytes of the hypertrophied myocardium of untreated SHR in accordance to the overall results shown in Fig. 3. The increase in CSA was reversed by cariporide both with the low- and the high-dose treatment.

Fig. 5 shows length-tension curves of isolated left ventricular papillary muscles from untreated age-matched NT and SHR, and cariporide-treated (low- and high-dose) SHR rats. The muscles isolated from hypertensive hypertrophied hearts were stiffer than those from the normotensive animals (shift to the left in the length-tension curve). Chronic treatment with the NHE-1 inhibitor, in spite of its effects on $\mathrm{HW} / \mathrm{BW}$ ratio and cardiomyocyte size, did not return the stiffness to control levels at any dosage used. The collagen volume fraction in the left


Fig. 4. Representative microphotographs of the left ventricle of age-matched untreated NT (panel A), untreated SHR (panel B), high-dose cariporidetreated SHR (panel C) and low-dose cariporide-treated SHR rats (panel D). Cells were stained with a trichromic technique including Gill haematoxylin, xylidine ponceau and picric acid. Magnification $1000 \times$. Bar $=10 \mu \mathrm{m}$. 


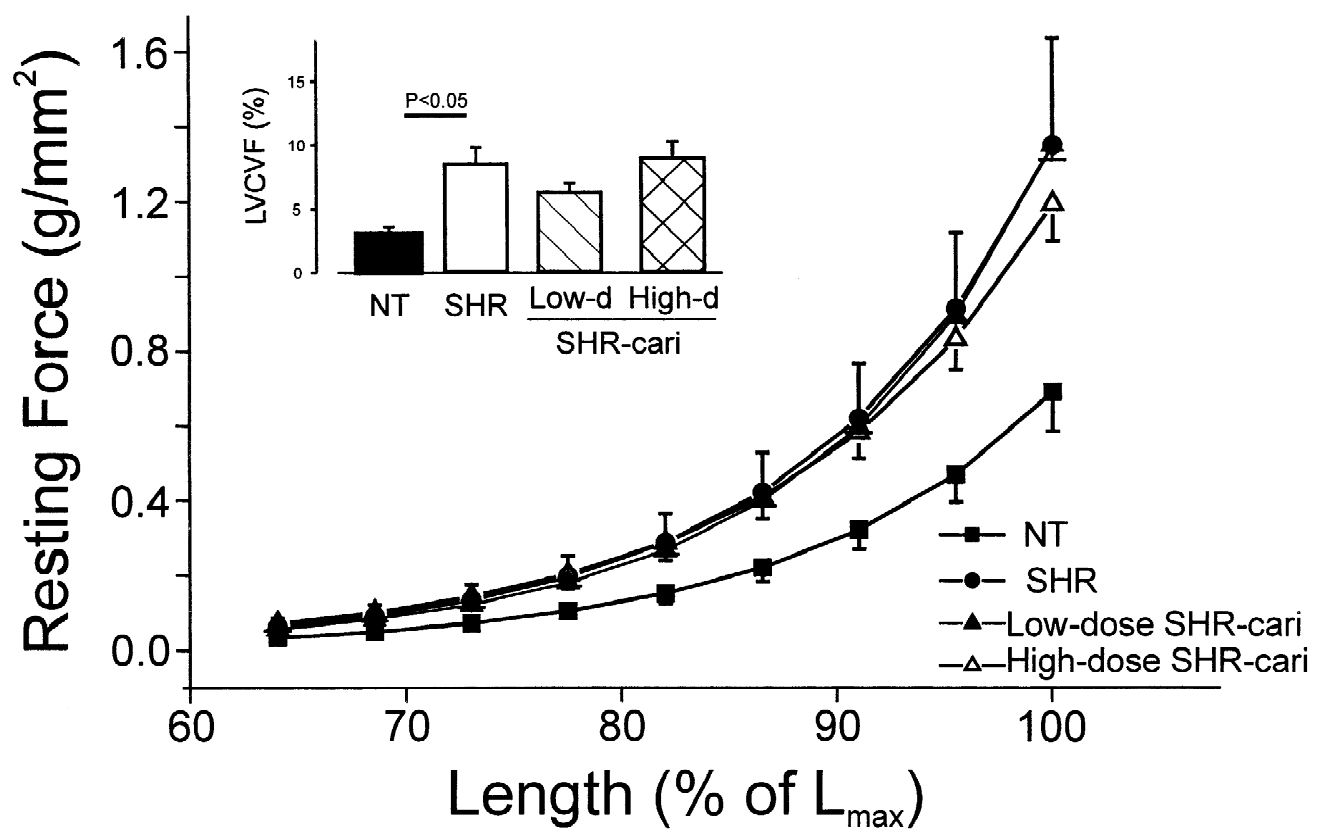

Fig. 5. Length-tension relationship in left ventricle papillary muscles. The muscles were isolated from age-matched untreated NT $(\boldsymbol{\square}, n=8)$, untreated $\operatorname{SHR}(\bullet, n=8)$, low-dose cariporide-treated SHR $(\boldsymbol{\Lambda}, n=5)$ and high-dose cariporide-treated SHR $(\Delta, n=7)$. Papillary muscles from untreated SHR showed increased stiffness compared to NT rats. NHE-1 inhibition did not reduce the increased stiffness in SHR despite reduction in cardiac hypertrophy. Inset: shows collagen volume fraction in left ventricle (LVCVF) determined in untreated NT (closed bar, $n=4)$, untreated SHR (open bar, $n=4)$, low-dose cariporide-treated SHR (hatched bar, $n=5$ ) and high-dose cariporide-treated SHR (cross-hatched bar, $n=4$ ) rats are shown. The NHE-1 inhibition did not modify the increased fibrosis of the SHR myocardium.

ventricle (LVCVF) of age-matched untreated NT, untreated SHR, and the low- and high-dose cariporide-treated SHR is shown in Fig. 5, inset. The increase in LVCVF in the myocardium of the SHR is in agreement with previous reports $[19,20]$. The treatment with the NHE-1 inhibitor did not result in a significant change of the LVCVF.

Since the increase in serum levels of carboxyterminal propeptide of procollagen type I (PIP), a marker of collagen type I synthesis, has been shown to correlate with

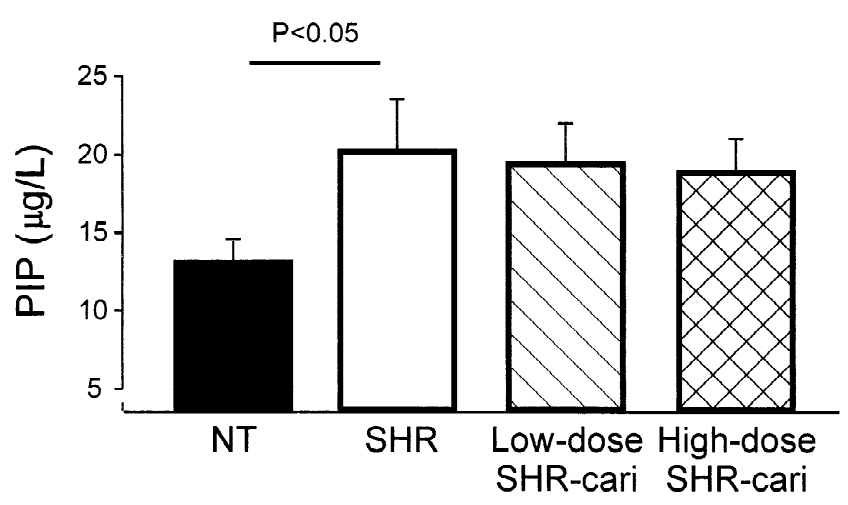

Fig. 6. Serum levels of the procollagen type I peptide (PIP) in agematched untreated NT (closed bar, $n=9$ ) and untreated SHR (open bar, $n=12$ ), low-dose cariporide-treated SHR (hatched bar, $n=5$ ) and highdose cariporide-treated SHR (cross-hatched bar, $n=5$ ) rats are shown. The increased levels of PIP in SHR were not modified by the NHE-1 inhibition. the extent of myocardial fibrosis in SHR [20], serum PIP levels were determined in the normotensive, hypertensive and hypertensive rats treated with cariporide and these data are shown in Fig. 6. The increased levels of PIP in the blood of SHR compared to NT rats are in agreement with the previously reported value [20]. Neither low-dose nor high-dose cariporide treatment resulted in a significant change of PIP levels in SHR. These data are in agreement with the lack of regression of the increased stiffness and LVCVF. Again, the possibility that it would be necessary to have a longer duration of the treatment to obtain a decrease in PIP should be considered, taking into account that 20 weeks of treatment are required to decrease the PIP levels with an ACE inhibitor [20].

\section{Discussion}

This is the first report to document regression of myocardial hypertrophy by an orally administered specific NHE-1 inhibitor. This effect was achieved with a slight decrease of $\sim 6 \mathrm{mmHg}$ from pretreatment $\mathrm{SBP}$ value whereas a similar reduction of hypertrophy induced by nifedipine was accompanied by a decrease in arterial pressure of $\sim 36 \mathrm{mmHg}$. Two recent reports, however, called attention to the effect of in vivo NHE inhibition by cariporide on cellular growth. The first one describes the 
regression of vascular hypertrophy in the diabetic rat [21], and the second one shows the prevention of cardiomyocyte hypertrophy after myocardial infarction [22] at a dosage similar to the lower one used in our study.

Both the dosage and the duration of the cariporide treatment used in the present experiments deserve some consideration. A wide range of cariporide dosages have been used by different investigators, and ours were either lower than [21,23] or similar to [22,24] those of some previous reports. Although we did not measure circulating cariporide levels, the effect to regress and prevent further increase in cardiac hypertrophy was evident. In addition, the fact that similar effects were obtained with the two doses of cariporide used shows that this chronic treatment was close to, if not at, the maximal effect for decreasing cardiomyocyte size. The possibility of tissue accumulation of the NHE-1 inhibitor should also be considered.

The regression of the myocyte size was not accompanied by a significant regression of cardiac fibrosis over the 1-month study. The histological staining technique used does not distinguish between the different collagen fractions. However, the increased stiffness detected in the papillary muscles of SHR suggests the possibility of alterations in collagen cross-linking [25]. The duration of the treatment in this study was too short as to ascertain whether or not this pharmacological intervention would also decrease fibrosis, or induce a total regression of heart weight. Results obtained with different antihypertensive treatments on hypertrophied myocardium suggest that myocyte and nonmyocyte cells grow independently of each other and that myocyte size declines more rapidly than the involution of collagen [26]. Actually, whereas regression of myocyte size was obtained after 6 weeks of antihypertensive treatment, no regression of collagen was observed at that time [27]. Since previous studies have shown that regression of collagen and normalization of PIP levels can be achieved with extended treatment with other pharmacological interventions $[19,20]$, it appears that longer treatments would be necessary to establish whether a similar result could happen with cariporide. In this regard, ongoing experiments blocking NHE-1 for longer periods will allow us to learn whether or not this novel pharmacological intervention is able to decrease myocardial fibrosis and stiffness.

In order to discuss the possible mechanisms by which the chronic blockade of the NHE-1 may induce the regression of cardiac hypertrophy it is necessary to review the available data linking the enhanced activity of the NHE-1 to hypertrophic mechanisms. In the first place, an enhanced activity of the NHE-1 in the hypertrophied myocardium of the SHR has been reported [8,9]. Also, the regression of the cardiac hypertrophy induced by ACE inhibition was accompanied by normalization of NHE activity [10]. We also reported that NHE activation is an early signal elicited by the stretch of papillary muscles [1] that results from an autocrine/paracrine release of Ang II and ET [2-4]. A role for endogenous ET-1 locally produced and secreted by cardiomyocytes in the mechanism of Ang II-induced cardiac hypertrophy was proposed [28].

Stretch-induced release of Ang II and its autocrine link with myocyte hypertrophy was demonstrated by Sadoshima et al. [5] in isolated neonatal rat cardiac cells. In the same preparation, the group of Yamazaki showed that whereas NHE inhibition partially attenuated the increase in protein synthesis and MAP kinases activation promoted by stretch [7,29], it did not inhibit Ang II- and ET-1-induced activation of these kinases [7]. The involvement of MAP kinase and $\mathrm{p} 90^{r s k}$-dependent phosphorylation of the NHE in ET-1-induced stimulation of the antiporter was recently demonstrated by Moor and Fliegel [30].

We may speculate that if an enhanced activity of the NHE were maintaining an augmented $\mathrm{Na}_{\mathrm{i}}^{+}$concentration, blockade of NHE-1 would restore the $\mathrm{Na}_{i}^{+}$concentration and thereby, blunt $\mathrm{Ca}^{2+}$ influx through $\mathrm{Na}^{+} / \mathrm{Ca}^{2+}$ exchange. The notion that the increase in $\mathrm{Ca}_{\mathrm{i}}^{2+}$ is a primary signal for cardiac hypertrophy is supported by numerous studies [31-35]. Yamazaki et al. [36] suggested two independent hypertrophic pathways, one elicited by mechanical stretch and linked to NHE activation and another linked to Ang II or ET-1 without involvement of NHE activation. Our experiments in multicellular preparations from adult myocardium would support the contention that the Ang II, ET-1 and NHE are all steps of a single chain of events beginning with the release of Ang II and ending with the increase in $\mathrm{Na}_{\mathrm{i}}^{+}$due to NHE activation by ET-1.

The notion that an increase in intracellular $\mathrm{Na}_{i}^{+}$leads to a secondary increase in $\mathrm{Ca}_{\mathrm{i}}^{2+}$ is supported by several studies $[2,37,38]$. Furthermore, the link between $\mathrm{Na}^{+}$ influx, activation of PKC- $\delta$ and $\varepsilon$, and hypertrophy has been previously suggested [39]. Although stretch-sensitive channels are apparently good candidates to be analyzed as regards their possible role in the mechano-transduction mechanism, they do not seem to play any role in this pathway $[5,7]$.

In summary, we report a regression of the cardiomyocyte hypertrophy in SHR after 1-month of NHE-1 blockade. This regression of myocyte size occurred without significant changes in the augmented serum PIP levels, LVCVF and myocardial stiffness. These novel findings may help to find the way to develop new therapeutic strategies for the treatment of cardiac hypertrophy.

\section{Acknowledgements}

This study was supported in part by grant Ramón Carrillo-Arturo Oñativia, Ministerio de Salud de la Nación, Argentina, to H.E. Cingolani. The technical assistance of Laura Paoli and Rosa Villegas is acknowledged. 


\section{References}

[1] Cingolani HE, Alvarez BV, Ennis IL, Camilión de Hurtado MC. Stretch-induced alkanization of feline papillary muscle. An autocrine-paracrine system. Circ Res 1998;83:775-779.

[2] Alvarez BV, Pérez NG, Ennis IL, Camilión de Hurtado MC, Cingolani HE. Mechanisms underlying the increase in force and $\mathrm{Ca}^{2+}$ transient that follow stretch of cardiac muscle. A possible explanation of the Anrep effect. Circ Res 1999;85:716-722.

[3] Camilión de Hurtado MC, Alvarez BV, Pérez NG, Ennis IL, Cingolani HE. Angiotensin II activates $\mathrm{Na}^{+}$-independent $\mathrm{Cl}^{-}{ }_{-}$ $\mathrm{HCO}_{3}^{-}$exchange in ventricular myocardium. Circ Res 1998;82:473481.

[4] Camilión de Hurtado MC, Alvarez BV, Ennis IL, Cingolani HE. Stimulation of myocardial $\mathrm{Na}^{+}$-independent $\mathrm{Cl}^{-}-\mathrm{HCO}_{3}^{-}$exchanger by endogenous endothelin. Circ Res 2000;86:622-626.

[5] Sadoshima J, Xu Y, Slayter HS, Izumo S. Autocrine release of angiotensin II mediates stretch-induced hypertrophy of cardiac myocytes in vitro. Cell 1993;75:977-984.

[6] Yamazaki T, Komuro I, Kudoh S et al. Endothelin-1 is involved in mechanical stress-induced cardiomyocyte hypertrophy. J Biol Chem 1996;271:3221-3228.

[7] Yamazaki T, Komuro I, Kudoh S et al. Role of ion channels and exchangers in mechanical stretch-induced cardiomyocyte hypertrophy. Circ Res 1998;82:430-437.

[8] Pérez NG, Alvarez BV, Camilión de Hurtado MC, Cingolani HE. $\mathrm{pH}_{\mathrm{i}}$ regulation in myocardium of the spontaneously hypertensive rat. Compensated enhanced activity of the $\mathrm{Na}^{+}-\mathrm{H}^{+}$exchanger. Circ Res 1995;77:1192-1200.

[9] Schussheim AE, Radda GK. Altered $\mathrm{Na}^{+}-\mathrm{H}^{+}$exchange activity in the spontaneously hypertensive perfused rat heart. J Mol Cell Cardiol 1995;27:1475-1481.

[10] Ennis IL, Alvarez BV, Camilión de Hurtado MC, Cingolani HE. Enalapril induces regression of cardiac hypertrophy and normalization of $\mathrm{pH}_{\mathrm{i}}$ regulatory mechanisms. Hypertension 1998;31:961-967.

[11] Buñag RA. Validation in awake rats of a tail-cuff method for measuring systolic pressure. J Appl Physiol 1973;34(2):279-282.

[12] Bradbury P, Gordon KC. Connective tissues and stains. In: Bancroft JD, Edimburg SA, editors, 3rd ed, Theory and practice of histological techniques, London: Churchill Livingstone, 1990, pp. 119-142.

[13] Kranzhofer R, Schirmer J, Schomig A et al. Suppression of neointimal thickening and smooth muscle cell proliferation after arterial injury in the rat by inhibitors of $\mathrm{Na}^{+}-\mathrm{H}^{+}$exchange. Circ Res 1993;73:264-268.

[14] Motz W, Ploeger M, Ringsgwandl G et al. Influence of nifedipine on ventricular function and myocardial hypertrophy in spontaneously hypertensive rats. J Cardiovasc Pharmacol 1983;5:55-61.

[15] Motz W, Strauer BE. Left ventricular function and collagen content after regression of hypertensive hypertrophy. Hypertension 1989;13:43-50.

[16] Kimpara T, Okabe M, Nishimura $H$ et al. Ultrastructural changes during myocardial hypertrophy and its regression: long-term effects of nifedipine in adult spontaneously hypertensive rats. Heart Vessels 1997;12:143-151.

[17] Brilla CG. Regression of myocardial fibrosis in hypertensive heart disease: diverse effects of various antihypertensive drugs. Cardiovasc Res 2000;46:324-331.

[18] López B, Querejeta R, Varo N et al. Usefulness of serum carboxyterminal propeptide of procollagen type $I$ in assessment of the cardioreparative ability of antihypertensive treatment in hypertensive patients. Circulation 2001;104:286-291.

[19] Brilla CG, Janicki JS, Weber KT. Cardioreparative effects of lisinopril in rats with genetic hypertension and left ventricular hypertrophy. Circulation 1991;83:1771-1779.

[20] Díez J, Panizo A, Gil MJ et al. Serum markers of collagen type I metabolism in spontaneously hypertensive rats. Relation to myocardial fibrosis. Circulation 1996;93:1026-1032.
[21] Jandeleit-Dahm K, Hannan KM, Farrelli CA et al. Diabetes-induced vascular hypertrophy is accompanied by activation of $\mathrm{Na}^{+}-\mathrm{H}^{+}$ exchange and prevented by $\mathrm{Na}^{+}-\mathrm{H}^{+}$exchange inhibition. Circ Res 2000;87:1133-1140.

[22] Yoshida H, Karmazyn M. $\mathrm{Na}^{+} / \mathrm{H}^{+}$exchange inhibition attenuates hypertrophy and heart failure in 1-wk postinfarction rat myocardium. Am J Physiol Heart Circ Physiol 2000;278:H300-H304.

[23] Kusumoto K, Haist JV, Karmazyn M. $\mathrm{Na}^{+} / \mathrm{H}^{+}$exchange inhibition reduces hypertrophy and heart failure after myocardial infarction in rats. Am J Physiol Heart Circ Physiol 2001;280:H738-H745.

[24] Humphreys RA, Haist JV, Chakrabarti S et al. Orally administered NHE1 inhibitor cariporide reduces acute responses to coronary occlusion and reperfusion. Am J Physiol Heart Circ Physiol 1999;276:H749-H757.

[25] Norton GR, Tsotetsi J, Trifunovic B et al. Myocardial stiffness is attributed to alterations in cross-linked collagen rather than total collagen or phenotypes in spontaneously hypertensive rats. Circulation 1997;96:1991-1998.

[26] Weber KT, Brilla CG. Pathological hypertrophy and cardiac interstitium. Fibrosis and renin-angiotensin-aldosterone system. Circulation 1991;83:1849-1865.

[27] Sen S, Tarazi RC, Bumpus FM. Biochemical changes associated with development and reversal of cardiac hypertrophy in spontaneously hypertensive rats. Cardiovasc Res 1976;10(2):254-261.

[28] Ito $\mathrm{H}$, Hirata $\mathrm{Y}$, Adachi $\mathrm{S}$ et al. Endothelin-1 is an autocrine/ paracrine factor in the mechanism of angiotensin II-induced hypertrophy in cultured rat cardiomyocytes. J Clin Invest 1993;92:398-403.

[29] Takewaki S-I, Kuro-o M, Hiroi $\mathrm{Y}$ et al. Activation of $\mathrm{Na}^{+}-\mathrm{H}^{+}$ antiporter (NHE-1) gene expression during growth, hypertrophy and proliferation of the rabbit cardiovascular system. J Mol Cell Cardiol 1995;27:729-742.

[30] Moor AN, Fliegel L. Protein kinase-mediated regulation of the $\mathrm{Na}^{+} / \mathrm{H}^{+}$exchanger in the rat myocardium by mitogen-activated protein kinase-dependent pathways. J Biol Chem 1999;274(33):22985-22992.

[31] Marban E, Koretsune Y. Cell calcium, oncogenes and hypertrophy. Hypertension 1990;15:652-658.

[32] Eguchi S, Matsumoto T, Motley ED, Utsunomiya H, Inagami $\mathrm{T}$. Identification of an essential signaling cascade for mitogen-activated protein kinase activation by angiotensin II in cultured rat vascular smooth muscle cells. J Biol Chem 1996;271:14169-14175.

[33] Zou Y, Komuro I, Yamazaki T et al. Protein kinase C, but not tyrosine kinases of Ras, plays a critical role in angiotensin IIinduced activation of Raf-1 kinase and extracellular signal regulated protein kinases in cardiac myocytes. J Biol Chem 1996;271:3359233597.

[34] Dolmetsch RE, Lewis RS, Goodnow CC, Healy JI. Differential activation of transcription factors induced by $\mathrm{Ca}^{2+}$ response amplitude and duration. Nature 1997;386:855-858.

[35] Mende U, Kagen A, Cohen J et al. Transient cardiac expression of constitutively activated $\mathrm{G} \alpha_{\mathrm{q}}$ leads to cardiac hypertrophy and dilated cardiomyopathy by calcineurin-dependent and independent pathways. Proc Natl Acad Sci USA 1998;95:13893-13898.

[36] Yamazaki T, Komuro I, Yazaki Y. Role of the renin-angiotensin system in cardiac hypertrophy. Am J Cardiol 1999;83:53-57.

[37] Pérez NG, Camilión de Hurtado MC, Cingolani HE. Reverse mode of the $\mathrm{Na}^{+} / \mathrm{Ca}^{2+}$ exchange following myocardial stretch. Underlying mechanism of the slow force response. Circ Res 2001;88:376382.

[38] Terada H, Hayashi H, Satoh H, Katoh H, Yamazaki N. Simultaneous measurement of $\left[\mathrm{Na}^{+}\right]_{\mathrm{i}}$ and $\mathrm{Ca}^{2+}$ transients in isolated myocyte: effects of strophantidin. Biochem Biophys Res Commun 1994;203:1050-1056.

[39] Hayasaki-Kajiwara Y, Kitano Y, Iwasaki $\mathrm{T}$ et al. $\mathrm{Na}^{+}$influx via $\mathrm{Na}^{+} / \mathrm{H}^{+}$exchange activates protein kinase $\mathrm{C}$ isozymes $\delta$ and $\varepsilon$ in cultured neonatal rat cardiac myocytes. J Mol Cell Cardiol 1999;31:1559-1575. 